\title{
The Effect of Dopamine Agonists on Adaptive and Aberrant Salience in Parkinson's Disease
}

\author{
Helga Nagy ${ }^{1,2}$, Einat Levy-Gigi ${ }^{3}, Z^{5}$ suzsanna Somlai ${ }^{4}$, Annamária Takáts', Dániel Bereczki' and \\ Szabolcs Kéri*,5,6 \\ 'Department of Neurology, Semmelweis University, Budapest, Hungary; ${ }^{2}$ National Institute for Medical Rehabilitation, Budapest, Hungary; \\ ${ }^{3}$ Center for Molecular and Behavioral Neuroscience, Rutgers University, Newark, NJ, USA; ${ }^{4}$ Department of Psychiatry, Semmelweis University, \\ Budapest, Hungary; ${ }^{5}$ Faculty of Medicine, Department of Physiology, University of Szeged, Szeged, Hungary; ${ }^{6}$ National Psychiatry Center, \\ Budapest, Hungary
}

Clinical evidence suggests that after initiation of dopaminergic medications some patients with Parkinson's disease (PD) develop psychotic symptoms, such as hallucinations and delusions. Here, we tested the hypothesis that the neurocognitive basis of this phenomenon can be defined as the formation of arbitrary and illusory associations between conditioned stimuli and reward signals, called aberrant salience. Young, never-medicated PD patients and matched controls were assessed on a speeded reaction time task in which the probe stimulus was preceded by conditioned stimuli that could signal monetary reward by color or shape. The patients and controls were re-evaluated after 12 weeks during which the patients received a dopamine agonist (pramipexole or ropinirole). Results indicated that dopamine agonists increased both adaptive and aberrant salience in PD patients, that is, formation of real and illusory associations between conditioned stimuli and reward, respectively. This effect was present when associations were assessed by means of faster responding after conditioned stimuli signaling reward (implicit salience) and overt rating of stimulus-reward links (explicit salience). However, unusual feelings and experiences, which are subclinical manifestations of psychotic-like symptoms, were specifically related to irrelevant and illusory stimulus-reward associations (aberrant salience) in PD patients receiving dopamine agonists. The learning of relevant and real stimulus-reward associations (adaptive salience) was not related to unusual experiences. These results suggest that dopamine agonists may increase psychotic-like experiences in young patients with PD, possibly by facilitating dopaminergic transmission in the ventral striatum, which results in aberrant associations between conditioned stimuli and reward.

Neuropsychopharmacology (2012) 37, 950-958; doi:10.1038/npp.20 I I.278; published online I6 November 20 I I

Keywords: Parkinson's disease; dopamine agonists; psychosis; reward; salience; cognition

\section{INTRODUCTION}

Although dopaminergic deficit is a hallmark of Parkinson's disease (PD), loss of this neurotransmitter is not evenly distributed in the striatum. Specifically, there is a more pronounced reduction of dopamine in the dorsal than in the ventral striatum (Kish et al, 1988). Therefore, therapies that restore dopamine level in the dorsal striatum result in dopamine 'overdose' in the ventral striatum, which may lead to impaired performance on some cognitive tasks (Gotham et al, 1988; Cools et al, 2001, 2003; Shohamy et al, 2006; Jahanshahi et al, 2010; MacDonald et al, 2011) and in

*Correspondence: Dr S Kéri, Department of Physiology, University of Szeged, Dóm sq. 10, Szeged H6720, Hungary, Tel: + 36-20-448-3530, Fax: + 36-62-545-842, E-mail: keri.szabolcs.gyula@med.u-szeged.hu or szkeri2000@yahoo.com

Received 22 August 2011; revised 4 October 2011; accepted 5 October 2011 some cases psychotic symptoms, including hallucinations and delusions (McGowan et al, 2004; Mehler-Wex et al, 2006; Maia and Frank, 2011). At the same time, there is evidence that dopaminergic therapy enhances learning from reward signals and decreases learning from punishment signals in PD (Frank et al, 2004, 2007; Cools et al, 2006; Bódi et al, 2009; Graef et al, 2010; Kobayakawa et al, 2010), and the ventral striatum has a crucial role in reinforcement learning (Yin and Knowlton, 2006).

Conditioned stimuli associated with reward elicit phasic dopamine release in the striatum relative to stimuli that fail to predict reward (Schultz, 2007), which is important in human reinforcement learning (Pessiglione et al, 2006). It has also been shown that conditioned stimuli increase the speed of responses, and this effect is mediated by dopaminergic signals in the ventral striatum (Wyvell and Berridge, 2000). This phenomenon is called motivational or incentive salience, as a formerly neutral stimulus becomes 
associated with a motivational value that guides purposeful behavior (Berridge, 2007). King et al (1984) postulated that abnormal dopaminergic signals lead to chaotic and arbitrary stimulus-reward associations, which may be related to disorganized and psychotic behavior in some PD patients receiving dopaminergic drugs. This hypothesis of aberrant salience and the role of dopamine were emphasized in the context of psychiatric disorders such as schizophrenia (Kapur, 2003; Miller, 1993; Roiser et al, 2009; Schmidt and Roiser, 2009; Shaner, 1999). Housden et al (2010) suggested that excessive dopaminergic transmission results in a strong preference for immediate over future rewards in PD patients with impulsive-compulsive behaviors (eg, punding, hyper-sexuality, pathological gambling, compulsive shopping, and binge eating), but its relationship with psychotic-like symptoms is less clear.

Despite the rich theoretical background, no longitudinal study has been performed to investigate the effect of dopaminergic drugs on adaptive and aberrant salience in patients with PD. To achieve this aim, we recruited young, never-medicated patients with PD and matched healthy controls. We followed up the patients after the initiation of dopamine agonists (pramipexole and ropinirole) in order to study the effect of these drugs on adaptive and aberrant salience, and on subjective feelings and experiences related to psychosis.

Participants completed a speeded reaction time task in which the reward after the response was signaled by conditioned stimuli preceding the probe stimulus (Figure 1) (Roiser et al, 2009; Schmidt and Roiser, 2009).

We hypothesized that dopaminergic therapy increases adaptive salience, and therefore patients will show a more pronounced conditioning effect after initiation of dopaminergic medications. The critical question was how dopamine agonists affect aberrant salience, that is, the effect of task-irrelevant conditioned stimuli on responses and subjective ratings of salience. We predicted that in the therapeutic range pramipexole and ropinirole have no significant effect on aberrant salience, given that the majority of patients receiving this treatment do not develop psychotic symptoms.

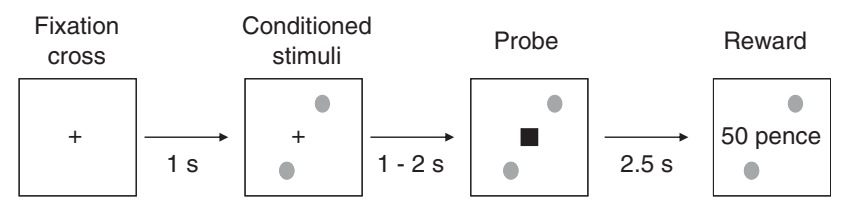

Figure I The salience attribution test. Participants were asked to press a button as quickly as possible when the probe stimulus (black square) appeared on the computer screen. The probe was preceded by conditioned stimuli that could signal reward. Either color or shape predicted reward. For example, green signaled reward regardless of shape (circle or triangle), and therefore shape was the task-irrelevant dimension. Adaptive salience means that participants explicitly rate reward more likely and implicitly respond faster when conditioned stimuli predicting reward precede the probe (eg, color green). In the case of aberrant salience, a similar phenomenon can be observed for the task-irrelevant stimulus dimension (eg, faster responses in the case of circle relative to triangle despite the fact that shape did not predict reward). In this case, association between conditioned stimuli and reward is illusory and not based on real contingencies.

\section{MATERIALS AND METHODS}

\section{Participants}

We recruited 20 newly diagnosed, young patients with idiopathic PD who had never received dopaminergic medications. Patients were diagnosed and assessed by trained neurologists and psychiatrists who were naïve to the aim of the study. All patients met the UK Parkinson's Disease Society Brain Bank Clinical Diagnostic Criteria (Hughes et al, 1992). The PD patients were compared with 20 matched healthy volunteers. After baseline testing in unmedicated state, the patients started dopamine agonist therapy and were followed up for 12 weeks (pramipexole: $n=12$, mean dose at follow-up: $4.0 \mathrm{mg} /$ day, range: $2.0-$ $6.0 \mathrm{mg} /$ day; ropinirole: $n=8$, mean dose at follow-up: $8.5 \mathrm{mg} /$ day, range: $4.0-11.5 \mathrm{mg} /$ day). After this period, participants were re-evaluated. The patients also scored their overall subjective state using a -10 to +10 Likert-type scale $(-10$ : feeling very bad relative to the unmedicated state; +10 : feeling very good relative to the unmedicated state; 0: no changes after medication).

Neurological and psychiatric symptoms were assessed using the Hoehn-Yahr Scale (Hoehn and Yahr, 1967), the Unified Parkinson's Disease Rating Scale (UPDRS) (Lang and Fahn, 1989), the Hamilton Depression Rating Scale (HAM-D), the Hamilton Anxiety Rating Scale (HAM-A) (Mountjoy and Roth, 1982), and the Young Mania Rating Scale (YMRS) (Young et al, 1978). Impulsive-compulsive spectrum behavior was evaluated according to standardized criteria using structured interviews (Voon and Fox, 2007). Socioeconomic status was characterized by the Hollingshead Four-Factor Index (Cirino et al, 2002), and general intellectual functions were measured using the revised version of the Wechsler Adult Intelligence Scale (WAIS-R) (Wechsler, 1981). All scales were administered by trained experts who were blind to diagnosis, test performance, and medication status. The clinical and demographic data are presented in Table 1. All participants gave written informed consent and the study was approved by the institutional ethics board.

\section{Salience Attribution Test}

Stimuli were generated and presented using a VPC221 workstation (ViewSonic, Walnut, CA). Participants were asked to respond to a probe stimulus (a black square appearing on the computer screen) as quickly as possible by pressing a button. The probe stimulus was preceded by conditioned stimuli (colored shapes) predicting the probability of reward after the response (Figure 1). At the beginning of each trial, a fixation cross was presented. After $1 \mathrm{~s}$ the conditioned stimuli appeared at the top and bottom part of the screen, and remained there until the end of the trial. After an interval of $0.5-1.5 \mathrm{~s}$, the probe stimulus appeared on the screen and participants responded (Figure 1).

The duration of the probe stimulus was calculated for each participant during the training session preceding the main test. The practice trials were similar to the main test trials with the exception that conditioned stimuli were not presented and no reward was provided. Participants were 
Table I Clinical and Demographic Characteristics of Participants

\begin{tabular}{|c|c|c|}
\hline & Controls & $\begin{array}{l}\text { Parkinson's } \\
\text { patients }\end{array}$ \\
\hline Number of participants (male/female) & $20(14 / 6)$ & $20(14 / 6)$ \\
\hline Age (years) & $46.3(7.9)$ & $45.8(6.0)$ \\
\hline Education (years) & $14.2(6.3)$ & $14.3(7.0)$ \\
\hline $\begin{array}{l}\text { Time since onset of first } \\
\text { symptoms (months) }\end{array}$ & - & $20.3(9.6)$ \\
\hline Full-scale IQ (WAIS-R) & $109.6(10.5)$ & $107.4(12.9)$ \\
\hline Socioeconomic status (Hollingshead) & $37.9(15.2)$ & $38.5(16.9)$ \\
\hline \multirow[t]{4}{*}{ No. of patients in Hoehn-Yahr stage } & - & I.0: 3 \\
\hline & & 1.5: 2 \\
\hline & & $2: 14$ \\
\hline & & 2.5: 1 \\
\hline \multicolumn{3}{|l|}{ UPDRS total } \\
\hline Baseline & - & $34.4(9.8)$ \\
\hline Follow-up & - & $26.7(8.8)^{\mathrm{a}}$ \\
\hline \multicolumn{3}{|l|}{ UPDRS III (motor) } \\
\hline Baseline & - & $25.5(6.4)$ \\
\hline Follow-up & - & $20.5(7.5)^{\mathrm{b}}$ \\
\hline \multicolumn{3}{|l|}{ HAM-D } \\
\hline Baseline & $3.7(3.3)$ & $3.9(2.2)$ \\
\hline Follow-up & $3.7(2.9)$ & $3.8(2.3)$ \\
\hline \multicolumn{3}{|l|}{ HAM-A } \\
\hline Baseline & $3.0(2.5)$ & $3.4(1.9)$ \\
\hline Follow-up & $3.1(2.7)$ & $3.4(2.0)$ \\
\hline \multicolumn{3}{|l|}{ YMRS } \\
\hline Baseline & $1.0(0-2)$ & $1.0(0-4)$ \\
\hline Follow-up & $1.0(0-2)$ & $2.5(0-8)^{c}$ \\
\hline \multicolumn{3}{|l|}{ O-LIFE unusual experiences } \\
\hline Baseline & $9.0(3.5)$ & $8.5(3.0)$ \\
\hline Follow-up & $8.9(3.3)$ & $11.6(3.3)^{d}$ \\
\hline \multicolumn{3}{|l|}{ Cognitive disorganization } \\
\hline Baseline & $9.1(4.0)$ & $8.9(4.2)$ \\
\hline Follow-up & $9.1(4.1)$ & $8.6(4.2)$ \\
\hline \multicolumn{3}{|l|}{ Introvertive anhedonia } \\
\hline Baseline & $4.7(2.6)$ & $4.8(3.7)$ \\
\hline Follow-up & $4.9(2.8)$ & $4.0(3.3)$ \\
\hline \multicolumn{3}{|l|}{ Impulsive nonconformity } \\
\hline Baseline & $7.6(4.4)$ & $6.9(4.5)$ \\
\hline Follow-up & $7.2(4.7)$ & $7.1(4.6)$ \\
\hline
\end{tabular}

Abbreviations: HAM-A, Hamilton Anxiety Scale; HAM-D, Hamilton Depression Scale; O-LIFE, Oxford-Liverpool Inventory of Feelings and Experiences; UPDRS, Unified Parkinson's Disease Rating Scale; WAIS-R, Wechsler Adult Intelligence Scale, revised; YMRS, Young Mania Rating Scale.

Data are mean (SD) with the exception of YMRS where median and range are depicted.

${ }^{a} \mathrm{t}(38)=2.62, p=0.01$.

$b_{t}(38)=2.27, p=0.03$

${ }^{c} Z=-2.18, p=0.03$

$\mathrm{d}_{\mathrm{t}(38)}=-3.06, p=0.004$. simply asked to respond as quickly as possible when the probe stimulus appeared on the screen. At the beginning of the first practice session, the probe was exposed with variable durations (0.5-1.5 s, mean: $1 \mathrm{~s})$. Feedback was provided after $2 \mathrm{~s}$ ('Good' - responses before disappearance of the probe; 'Try to respond faster'-responses after disappearance of the probe; 'Too early' - responses before appearance of the probe). In the second practice session, the probe stimulus duration was set according to individual reaction time. We calculated the SD of the mean reaction time from the faster half of the trials for each participant. The mean probe stimulus duration was the mean reaction time from the first practice session. The maximum and minimum probe stimulus duration was calculated by adding or subtracting twice of the SD (mean reaction time $+/-2 \mathrm{SD}$ ). In the main test, the mean, minimum, and maximum probe stimulus durations were calculated from the data obtained from the second practice session.

The main test consisted of 100 trials (Figure 1). Reward (winning money) was delivered after $50 \%$ of trials. The likelihood of reward was signaled by the conditioned stimuli, which varied on two dimensions: color (green or red) and shape (circle or triangle). One of these dimensions was task-relevant. For example, one of the colors predicted reward on 40 out of the 50 trials $(80 \%)$ (eg, green circle and green triangle), whereas the other color signaled reward only on 10 out of the 50 trials (20\%) (eg, red circle and red triangle). The other dimension was task-irrelevant, so that 25 out of 50 trials $(50 \%)$ were rewarded for both types of stimuli (circle and triangle). Color and form did not differ regarding effectiveness of reward prediction, that is, there were no significant differences between conditions when color or form was the task-relevant dimension $(p>0.5)$.

On reinforced trials, participants won between 5 and 100 pence (exchanged to Hungarian Forints) depending on the latency of the response. On reinforced trials when participants made premature or late responses, they got 5 pence. If participants made a correct response (pressing the button after the onset but before the offset of the probe), the magnitude of the reward depended on the speed of the response:

$$
\mathrm{RM}=10+90 \times(\mathrm{RT}[\text { training }]-\mathrm{RT}[\text { trial }]) / 3 \times \mathrm{SD}
$$

RM: reward magnitude; RT (training): mean reaction time from the second training session; RT (trial): actual reaction time from the main test; SD: standard deviation of the mean reaction time from the faster half of the trials in the second training session.

The money was added to the total points of the participant, which was presented at the top of the screen. At the end of the test, participants were asked to indicate how they estimated the reward probability for each conditioned stimulus by clicking on a $10-\mathrm{cm}$ visual analog scale presented on the computer screen.

The current version of the test shows some differences compared with the paradigm of Roiser et al (2009). We used more simple conditioned stimuli without semantic properties, the number of blocks was different, and there were fewer trials. The main reason is that we intended to simplify and shorten the test to make it easier for patients who are less able or willing to stay on tasks. 
Implicit and explicit adaptive and aberrant salience can be characterized by reaction time and visual analog scale scores, respectively. Implicit adaptive salience is the difference between the reaction time on trials with low reward probability and that on trials with high reward probability collapsed across the task-irrelevant stimulus dimension. Larger differences indicate speeded responding on trials with high reward probability, which refers to implicit adaptive salience. Explicit adaptive salience is defined as the increase in rating on the visual analog scale for trials with high reward probability relative to trials with low reward probability collapsed across the task-irrelevant stimulus dimension.

Implicit and explicit aberrant salience are calculated in a similar way, but in this case reaction time and visual analog scale scores are determined by using the task-irrelevant stimulus dimension collapsed across the task-relevant stimulus dimension. Aberrant salience could be any deviation from equal reaction time or equal visual analog rating for the task-irrelevant stimulus dimension. In the case of each individual, 'high' and 'low' reward probability levels are determined according to the subjective response; 'high' is the type of irrelevant stimulus dimension to which the participant responded faster or rated higher on the visual analog scale (eg, responding faster or giving higher rates to red shapes as predicting reward despite the fact that color was the irrelevant dimension). Therefore, rewardpredictive value has no absolute meaning in the context of the irrelevant stimulus dimension (the 'high' level of the factor is always higher than the 'low' level of the factor by definition). A perfect rational learner would show zero aberrant salience.

In the data analysis, the dependent measures were reaction time, visual analog rating scores, and salience values.

\section{Assessment of Feelings and Experiences Related to Psychosis}

The Oxford-Liverpool Inventory of Feelings and Experiences (O-LIFE; Mason et al, 1995) questionnaire was used to assess everyday versions of feelings and experiences related to psychotic states. The instrument consists of 159 items with a dichotomous response format. O-LIFE measures four dimensions: Unusual Experiences (perceptual aberrations, magical thinking, and hallucinatory experiences), Introvertive Anhedonia (decreased pleasure and enjoyment from social and physical sources of pleasure, and avoidance of intimacy), Cognitive Disorganization (loosened association and poor concentration), and Impulsive Nonconformity (impulsive, eccentric, aggressive, and asocial traits).

\section{Data Analysis}

The STATISTICA 9.1 software (StatSoft, Tulsa) was used for data analysis. Normality of data distribution was checked with Kolmogorov-Smirnov tests. Levene's tests were used to check the homogeneity of variance. Repeated-measures analysis of variance (ANOVA) was performed to compare PD patients and controls on the salience attribution test, and to evaluate the effect of dopamine agonists at follow-up. The ANOVA was followed by Tukey Honestly Significant Difference (HSD) tests. Two-tailed $t$-tests were used for analysis of demographic data and scores on clinical scales. If the data violated normal distribution, Mann-Whitney $U$-tests were used. Pearson's product-moment correlation coefficients were calculated between salience attribution test variables and behavioral scales. The level of significance was set at $\alpha<0.05$.

\section{RESULTS}

\section{General Description of the Sample}

The clinical and demographic data are presented in Table 1. There were no significant differences between patients and controls regarding age, education, IQ, social-economic status, mood and anxiety, and baseline O-LIFE scores $(p>0.5)$. At the follow-up phase, the UPDRS scores were lower relative to the baseline. There was a slight but statistically significant increase in YMRS and O-LIFE unusual experiences scores in PD patients after initiation of dopamine agonist therapy (Table 1). Overall, the patients reported that at the follow-up phase their general subjective state was better than before therapy (mean: 5.4, $\mathrm{SD}=3.4)$.

\section{Reaction Time from the Salience Attribution Test}

We tested how medication affected reaction time on the salience test in patients with PD. For the relevant stimulus dimension, we conducted an ANOVA in which group (controls $v s$ PD) was the between-subject factor, and testing time (baseline $v s$ follow-up) and reward-predictive value of conditioned stimulus (high $v s$ low) were the within-subject factors.

This ANOVA revealed a significant main effect of predictive value $(\mathrm{F}(1,38)=38.55, p<0.0001)$. There were significant two-way interactions between testing time and group $(\mathrm{F}(1,38)=17.61, p=0.0002)$, and between testing time and predictive value $(\mathrm{F}(1,38)=12.30, p=0.001)$. Critically, we found a significant three-way interaction among group, testing time, and predictive value $(\mathrm{F}(1,38)=24.81, p<0.0001)$. All remaining main effects and interactions were not significant $(\mathrm{F}<3, p>0.05)$.

Post hoc tests indicated that in healthy controls reaction time was faster when the probe stimulus was preceded by conditioned stimuli with high predictive value relative to that with low predictive value $(p<0.05)$. However, this effect was present in PD patients only at follow-up testing when they received dopamine agonists $(p<0.001)$, but not in the unmedicated state $(p>0.5)$ (Figure 2). We also found that dopamine agonists decreased reaction time in patients with PD under the high-predicting condition $(p<0.001)$ but not under the low-predicting condition $(p=0.9)$. Controls showed similar values at both testing times $(p>0.5)$ (Figure 2).

For the irrelevant stimulus dimension, we conducted an ANOVA in which group (controls $v s \mathrm{PD}$ ) was the betweensubject factor and testing time (baseline $v s$ follow-up) was the within-subject factor. Reward-predictive value was not included in this analysis because it is hardly interpretable in the context of irrelevant stimulus dimension (by definition the 'high' stimulus will be associated with higher explicit rating and shorter reaction time than the 'low' stimulus). 

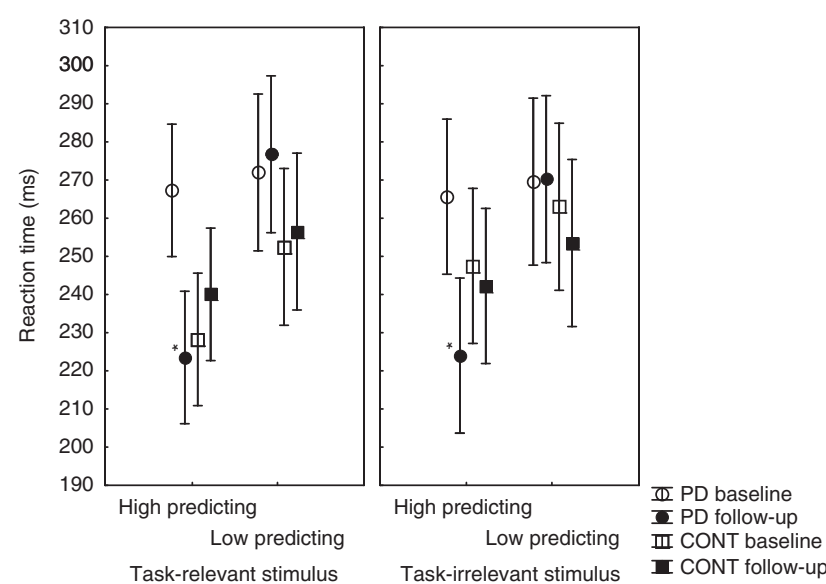

Figure 2 Mean reaction time in patients with PD $(n=20)$ and in healthy controls (CONT, $n=20$ ). The error bars indicate $95 \%$ confidence intervals. PD patients receiving dopamine agonists at follow-up showed faster responses for high-predicting stimuli relative to the unmedicated state $\left({ }^{*} p<0.0\right.$ I, Tukey HSD test). Note that reward-predictive value has no absolute meaning in the context of the irrelevant stimulus dimension (the 'high' level of the factor is always higher than the 'low' level of the factor by definition).

This ANOVA revealed a significant main effect of testing time $(\mathrm{F}(2,37)=7.89, p=0.002)$, and a two-way interaction between group and testing time $(\mathrm{F}(2,37)=7.53, p=0.002)$. Post hoc tests indicated that PD patient showed shorter reaction time at follow-up testing relative to baseline $(p<0.001)$ (Figure 2).

\section{Explicit Rating from the Salience Attribution Test}

We also investigated how medication affected explicit rating on the salience test in patients with PD. For the taskrelevant condition, we conducted an ANOVA in which group (controls $v s \mathrm{PD}$ ) was the between-subject factor, and testing time (baseline $v s$ follow-up) and reward-predictive value of conditioned stimuli (high vs low) were the withinsubject factors.

The ANOVA indicated significant main effects of group $(\mathrm{F}(1,38)=32.26, p<0.0001)$, testing time $(\mathrm{F}(1,38)=6.10$, $p=0.02)$, and predictive value $(\mathrm{F}(1,38)=83.68, p<0.0001)$. There were two-way interactions between group and testing time $(\mathrm{F}(1,38)=6.09, p=0.02)$, and group and predictive value $(\mathrm{F}(1,38)=9.63, p=0.004)$. The three-way interaction among group, testing time, and predictive value was significant $(\mathrm{F}(1,38)=5.03, p=0.03)$. All other results from the ANOVA were non-significant $(\mathrm{F}<3, p>0.05)$.

Post hoc tests indicated that dopamine agonists increased explicit rating scores in PD patients, but this effect was selective for high-predicting stimuli $(p<0.01)$. At baseline testing, PD patients showed lower rating scores for highpredicting stimuli relative to controls $(p<0.001)$. This difference remained significant at follow-up testing, but its magnitude was smaller $(p<0.05)$. In the case of lowpredicting stimuli, we did not observe differences between PD patients and controls, and controls showed similar values at both testing times $(p>0.5)$ (Figure 3$)$.

For the irrelevant stimulus dimension, we conducted an ANOVA in which group (controls vs PD) was the between-
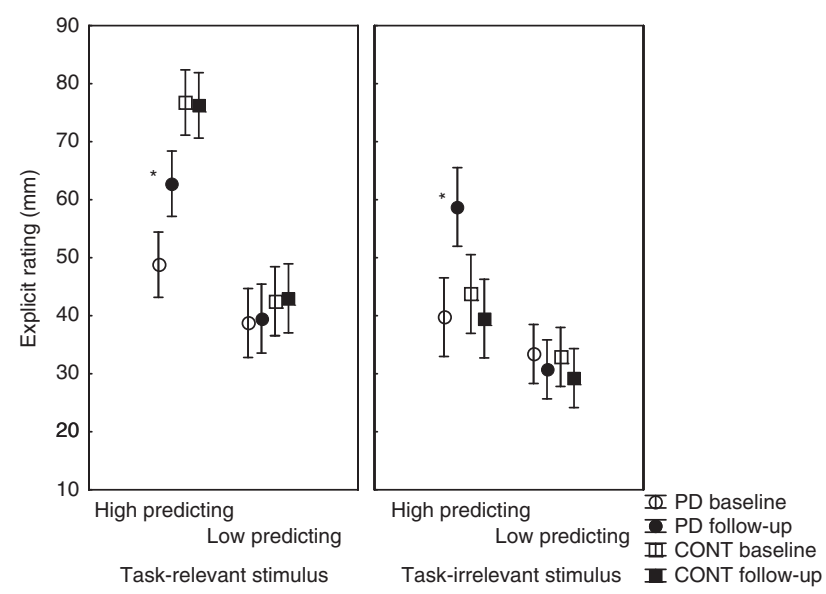

Figure 3 Mean visual analog rating scores in patients with PD $(n=20)$ and in healthy controls (CONT, $n=20$ ). The error bars indicate $95 \%$ confidence intervals. PD patients receiving dopamine agonists at follow-up showed increased rating scores for high-predicting stimuli relative to the unmedicated state (*p<0.0I, Tukey HSD test), but their score was still lower than that of the controls $(p<0.05)$. Note that reward-predictive value has no absolute meaning in the context of the irrelevant stimulus dimension (the 'high' level of the factor is always higher than the 'low' level of the factor by definition).

subject factor and testing time (baseline $v s$ follow-up) was the within-subject factor. There was a significant main effect of testing time $(\mathrm{F}(2,37)=10.39, p<0.001)$. The interaction between group and testing time was also significant $(\mathrm{F}(2,37)=11.50, p=0.0001)$.

According to the post hoc comparisons, PD patients showed a significantly increased rating score at follow-up testing relative to baseline $(p<0.001)$. Moreover, in the medicated state they showed higher rating scores relative to controls $(p<0.01)$ (Figure 3).

\section{Adaptive and Aberrant Salience Scores}

Table 2 shows adaptive and aberrant salience values calculated from reaction time (implicit salience) and visual analog rating scores (explicit salience). Table 3 presents the result from the ANOVAs and post hoc tests conducted on these salience scores. Overall, dopamine agonists increased all types of salience in PD (baseline $v s$ follow-up comparison), whereas values from the controls group showed stability in time.

\section{Premature Responses and Omissions}

There were no significant differences between PD patients and controls, or between testing sessions (baseline $v s$ follow-up) $(p>0.5)$ (Table 2).

\section{Relationship between Salience and Subjective Experiences Related to Psychosis}

In unmedicated patients with PD, there were no significant correlations among salience test measures and O-LIFE scores $(0.3>r>-0.3)$. By contrast, in PD patients receiving dopamine agonists, faster reaction time and higher subjective rating for task-irrelevant stimuli were associated 
Table 2 Salience Scores, Premature Responses, and Omissions

\begin{tabular}{|c|c|c|c|c|}
\hline \multirow[t]{2}{*}{ Measure } & \multicolumn{2}{|c|}{ Parkinson's patients $(n=20)$} & \multicolumn{2}{|c|}{ Controls $(n=20)$} \\
\hline & Baseline & Follow-up & Baseline & Follow-up \\
\hline Implicit adaptive salience & $4.7(24.5)$ & $53.2(27.8)$ & $24.3(36.8)$ & I 6.5 (32.7) \\
\hline Implicit aberrant salience & $4.0(10.4)$ & $46.3(47.3)$ & I5.5 (|6.1) & I $1.3(33.8)$ \\
\hline Explicit adaptive salience & $10.1(20.4)$ & $23.5(21.5)$ & $34.3(21.0)$ & $33.2(17.1)$ \\
\hline Omissions & $1.2(1.9)$ & $0.9(1.5)$ & I.I (1.7) & ।.3(2.0) \\
\hline
\end{tabular}

Data are mean (SD). Adaptive salience represents faster responding (implicit) or higher rating (explicit) for high reward predicting trials relative to low reward predicting trials. In the implicit condition adaptive salience is calculated as: mean reaction time on low reward predicting trials minus mean reaction time on high reward predicting trials. In the explicit condition adaptive salience is calculated as: visual analog rating scores on high reward predicting trials minus visual analog rating scores on low reward predicting trials. Implicit and explicit aberrant salience is calculated in a similar way, but in this case reaction time and visual analog scale measures are determined using the task-irrelevant stimulus dimension collapsed across the task-relevant stimulus dimension.

Table 3 Summary of ANOVA Results Conducted on Salience Scores

\begin{tabular}{|c|c|c|c|c|c|c|c|}
\hline & \multicolumn{2}{|c|}{$\begin{array}{l}\text { Main effect } \\
\text { of group }\end{array}$} & \multicolumn{2}{|c|}{$\begin{array}{l}\text { Main effect of } \\
\text { testing time }\end{array}$} & \multicolumn{2}{|c|}{$\begin{array}{l}\text { Group by testing } \\
\text { time interaction }\end{array}$} & \multirow[t]{2}{*}{ Post hoc comparison } \\
\hline & $\mathbf{F}$ & $p$ & $\mathbf{F}$ & $p$ & $\mathbf{F}$ & $p$ & \\
\hline Implicit adaptive salience & 1.18 & 0.28 & 12.98 & 0.001 & 24.81 & $<0.001$ & $\begin{array}{l}\mathrm{PD}[\text { med }]>\mathrm{PD}[\text { base }] \\
\mathrm{PD}[\text { med }]>\mathrm{CONT} \text { base, follow }] \\
\mathrm{PD}[\text { base }]=\mathrm{CONT}[\text { base }]=\mathrm{CONT}[\text { follow }]\end{array}$ \\
\hline Explicit adaptive salience & 9.63 & 0.004 & 3.72 & 0.06 & 5.03 & 0.03 & $\begin{array}{l}\mathrm{PD}[\text { med }]>\mathrm{PD}[\text { base }] \\
\mathrm{PD}[\text { med }]=\mathrm{CONT}[\text { base, follow }] \\
\mathrm{PD}[\text { base }]<\mathrm{CONT}[\text { base }]=\mathrm{CONT}[\text { follow }]\end{array}$ \\
\hline Explicit aberrant salience & 1.77 & 0.19 & 20.23 & $<0.001$ & 22.61 & $<0.001$ & $\begin{array}{l}\mathrm{PD}[\text { med }]>\mathrm{PD}[\text { base }] \\
\mathrm{PD}[\text { med }]>\text { CONT[base, follow }] \\
\mathrm{PD}[\text { base }]=\mathrm{CONT}[\text { base }]=\mathrm{CONT}[\text { follow }]\end{array}$ \\
\hline
\end{tabular}

Abbreviations: [base], unmedicated baseline; CONT, controls; [follow], re-tested at follow-up without medication (controls); [med], medicated at follow-up; PD, Parkinson's disease.

Post hoc comparisons: Tukey HSD, $p<0.05$.

with increased scores on the O-LIFE unusual experiences dimension obtained in the medicated state (reaction time: $r=-0.65, p<0.005$; rating: $r=0.57, p<0.05$ ). Reaction time and subjective rating for task-relevant stimuli did not correlate with O-LIFE scores $(r<0.1)$. The changes in aberrant salience scores and the change in O-LIFE (baseline $v s$ follow-up) were not significantly correlated $(r<0.2)$.

Finally, in controls we did not find a significant relationship between salience test measures and O-LIFE scores $(0.3>r>-0.3)$. Salience test results did not correlate with the YMRS scores $(r<0.1)$.

\section{Effects of Gender and Medication Type}

There were no significant differences between male and female patients $(p>0.5)$. Patients receiving pramipexole or ropinirole did not differ in salience test and O-LIFE scores $(p>0.1)$ (Figure 4$)$. The dose of dopamine agonists did not correlate with these measures $(-0.2<r<0.2)$.

\section{DISCUSSION}

Clinical studies suggest that psychotic symptoms are more frequent in PD patients receiving dopaminergic drugs than it was thought before (Weintraub and Burn, 2011). The severity and type of these symptoms depend on the duration and type of medication, the cognitive status of the patients, and pre-existing psychopathology (Ecker et al, 2009; Stoner et al, 2009; Verbaan et al, 2009). However, it has not been investigated whether dopamine agonists shift subjective feelings toward psychotic-like experiences in young, previously never-medicated patients with $\mathrm{PD}$, and how these unusual experiences are related to reward learning. Here, we showed that dopamine agonists facilitate not only adaptive and real stimulus-reward associations, but the emergence of illusory and arbitrary associations (aberrant salience). Importantly, faster reaction time and higher subjective rating for task-irrelevant stimuli, characterizing aberrant salience, correlated with sub-threshold psychotic-like feelings and experiences in PD patients 

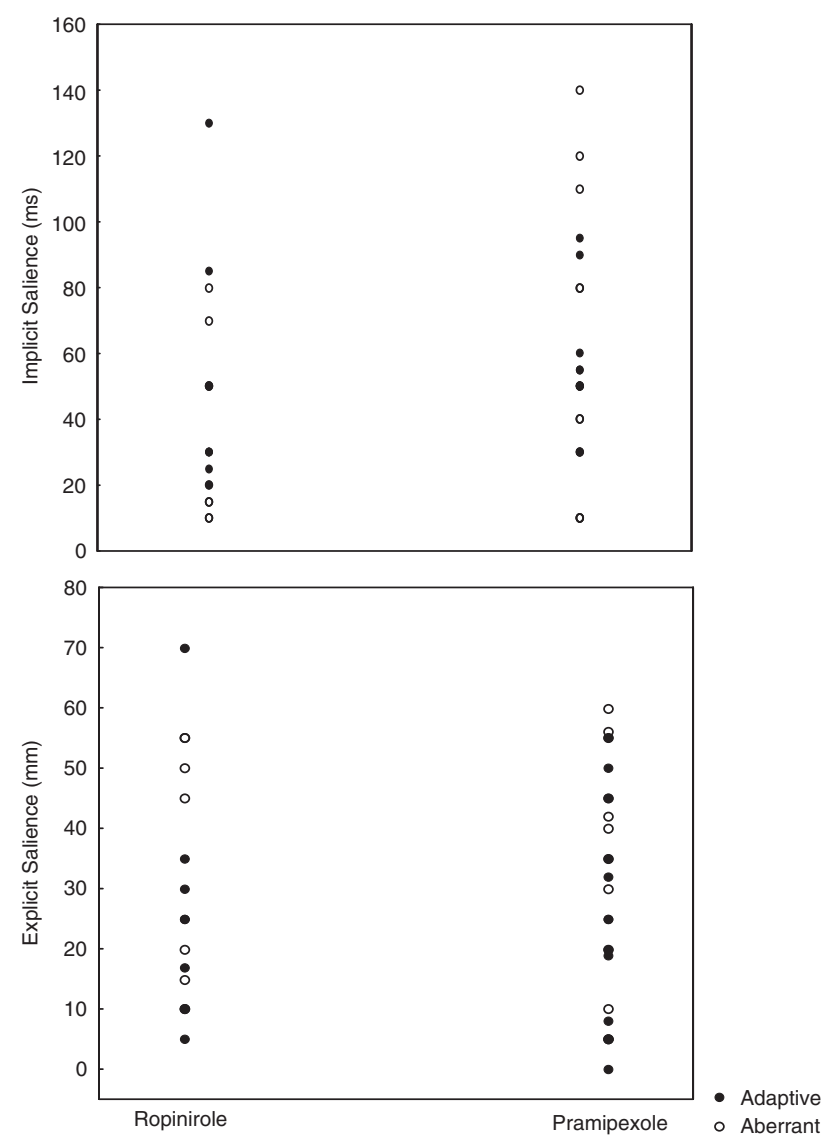

Figure 4 Scatterplot of explicit and implicit salience in patients with PD receiving ropinirole and pramipexole.

receiving dopamine agonists, which is against our initial hypothesis that in the therapeutic range these drugs are free from such effects. Moreover, after dopamine agonist administration PD patients showed higher scores relative to controls in the case of task-irrelevant but not in taskrelevant stimulus ratings, suggesting that information processing was biased toward aberrant salience (Figure 3). This effect was confirmed by aberrant salience scores (Tables 2 and 3).

However, it is essential to emphasize that none of the patients showed clinical psychosis, and the increase in unusual experiences after administration of dopamine agonists was moderate (27\%). The subjective well-being of the patients was increased after initiation of the therapy. Nevertheless, it is likely that patients who show marked unusual experiences are at higher risk for clinical psychosis. Independent from the salience scores, dopamine agonists also induced a slight increase in manic-like symptoms, as reflected by higher YMRS scores at the follow-up assessment.

Consistent with our results, Roiser et al (2009) showed that psychotic patients with delusions showed significantly greater aberrant salience than those without delusions. Intriguingly, in the study by Roiser et al (2009) aberrant salience also correlated with negative symptoms and O-LIFE introvertive anhedonia (reduced interest and social withdrawal). The authors interpreted this seemingly paradox phenomenon as a consequence of an abnormally high false-negative phasic dopamine signal. Specifically, Knutson et al (2004) demonstrated that amphetamine unexpectedly decreased the magnitude of phasic ventral striatal responses in response to conditioned stimuli predicting reward (false negatives), which could result in decreased motivational salience, leading to loss of volition and interest. On the other hand, however, amphetamine caused phasic ventral striatal activation for conditioned stimuli that did not predict reward (false positives), which may be related to aberrant salience.

Our results demonstrate that dopamine agonists may have a different effect compared with amphetamine, which releases dopamine from synaptic terminals; dopamine agonists increased both adaptive and aberrant stimulusreward associations, possibly by stimulation of $D_{2}$ and $D_{3}$ dopamine receptors in the ventral striatum (Gerlach et al, 2003; Winstanley et al, 2011). Indeed, the ventral striatum is implicated in general motivational salience, together with other key structures, including the midbrain dopaminergic centers and the prefrontal cortex (Roiser et al, 2010). Dopamine agonists may modulate the activation of this complex network (Ishibashi et al, 2011; Ye et al, 2011).

The effect of dopamine agonists may be a double-edged sword, as increased adaptive salience may improve activity and motivation, whereas aberrant salience may increase the risk of psychotic symptoms. Dopaminergic drugs also enhance memory for angry faces in PD (Subramanian et al, 2010), as well as action-effect binding, which is implicated in the subjective experience of controlling one's own actions (Moore et al, 2010). Enhanced representation of anger may contribute to paranoid ideations, leading to false interpretations of social signals (Kosaka et al, 2002), and the altered sense of controlling one's own actions may be also related to psychotic experiences, such as disrupted feeling of agency (Voss et al, 2010).

Cools et al (2010a) showed that attention was captured by bottom-up salient information to a greater extent in PD patients relative to healthy controls (but see also Mannan et al, 2008). Low dopamine levels in the striatum, together with potentially higher frontal dopamine levels, may contribute to increased resistance to distracting information in PD (Cools et al, 2010b). However, dopaminergic medication restores the balance between striatal and prefrontal dopamine levels, and hence may increase distractibility by bottom-up information, which can be interpreted as a form of enhanced salience. If this effect is exaggerated, distractibility may increase psychosis vulnerability. The prefrontal cortex is especially important in aberrant salience: participants who showed greater aberrant salience were characterized by enhanced prefrontal responses (Roiser et al, 2010). Dopamine agonists change value sensitivity in the orbitofrontal cortex and may impair negative reinforcement (van Eimeren et al, 2009). Further studies are needed to directly investigate the relationship between dopaminergic mechanisms in the prefrontal cortex and the striatum in the context of adaptive and aberrant salience.

The results of the present study extend the findings of Housden et al (2010) who investigated salience in chronic PD patients receiving both L-DOPA and dopamine agonists. These authors demonstrated that PD patients without impulsive-compulsive behavior showed impaired learning in the case of stimuli predicting reward with high 
probability, but PD patients with impulsive-compulsive behavior were able to learn such stimulus-reward associations. They did not find enhanced explicit aberrant salience, which was demonstrated in our medicated PD patients. Housden et al (2010) did not find significant correlation between O-LIFE unusual experiences and aberrant salience; instead, introvertive anhedonia was positively correlated with explicit aberrant salience and cognitive disorganization was positively correlated with implicit aberrant salience when all participants were included in the analysis. The correlation between implicit aberrant salience and cognitive disorganization may highlight another aspect of psychoticlike phenomena compared with unusual experiences. It is important that all PD patients showed higher O-LIFE unusual experiences and cognitive disorganization scores relative to controls, which was even more pronounced in PD patients with impulsive-compulsive symptoms who showed greater schizotypy on each O-LIFE dimension (Housden et al, 2010).

There are several differences between the study of Housden et al (2010) and the present investigation, including task structure (type of reward-predicting stimuli and number of experimental blocks) and the clinical characteristics of participants. In contrast to the patients of Housden et al (2010), we assessed young, nevermedicated patients with marked motor symptoms but short disease duration before and after dopamine agonist monotherapy, and our patients showed no impulsivecompulsive symptoms, depression, and anxiety.

Our study has two major limitations. First, healthy controls did not receive dopamine agonists, and therefore it remains to be elucidated whether the effect is specific for PD or not. Second, medication effects may be confounded by session order, given that dopamine agonist therapy always followed the unmedicated baseline testing. However, the finding that healthy controls did not show session effects suggests that the results are related to medications.

In conclusion, our findings have implications for both basic and clinical neuroscience. First, we demonstrated that dopamine agonists do not selectively enhance adaptive reward learning, but increase general salience signals leading to illusory and aberrant stimulus-reward associations. Second, our data highlight the importance of monitoring of subclinical psychotic-like feelings and experiences in patients receiving dopamine agonists in order to detect vulnerable persons and to prevent the development of clinical psychosis.

\section{ACKNOWLEDGEMENTS}

This study was supported by the Hungarian Research Fund (OTKA NF72488) and TAMOP-4.2.1.B-09/1/KMR.

\section{DISCLOSURE}

The authors declare no conflict of interest.

\section{REFERENCES}

Berridge KC (2007). The debate over dopamine's role in reward: the case for incentive salience. Psychopharmacology (Berl) 191: 391-431.
Bódi N, Kéri S, Nagy H, Moustafa A, Myers CE, Daw N et al (2009). Reward-learning and the novelty-seeking personality: a betweenand within-subjects study of the effects of dopamine agonists on young Parkinson's patients. Brain 132: 2385-2395.

Cirino PT, Chin CE, Sevcik RA, Wolf M, Lovett M, Morris RD (2002). Measuring socioeconomic status: reliability and preliminary validity for different approaches. Assessment 9: 145-155.

Cools R, Barker RA, Sahakian BJ, Robbins TW (2001). Enhanced or impaired cognitive function in Parkinson's disease as a function of dopaminergic medication and task demands. Cereb Cortex 11: 1136-1143.

Cools R, Barker RA, Sahakian BJ, Robbins TW (2003). L-Dopa medication remediates cognitive inflexibility, but increases impulsivity in patients with Parkinson's disease. Neuropsychologia 41: 1431-1441.

Cools R, Altamirano L, D'Esposito M (2006). Reversal learning in Parkinson's disease depends on medication status and outcome valence. Neuropsychologia 44: 1663-1673.

Cools R, Rogers R, Barker RA, Robbins TW (2010a). Top-down attentional control in Parkinson's disease: salient considerations. J Cogn Neurosci 22: 848-859.

Cools R, Miyakawa A, Sheridan M, D'Esposito M (2010b). Enhanced frontal function in Parkinson's disease. Brain 133: 225-233.

Ecker D, Unrath A, Kassubek J, Sabolek M (2009). Dopamine agonists and their risk to induce psychotic episodes in Parkinson's disease: a case-control study. BMC Neurol 9: 23.

Frank MJ, Seeberger LC, O'reilly RC (2004). By carrot or by stick: cognitive reinforcement learning in parkinsonism. Science 306: 1940-1943.

Frank MJ, Samanta J, Moustafa AA, Sherman SJ (2007). Hold your horses: impulsivity, deep brain stimulation, and medication in parkinsonism. Science 318: 1309-1312.

Gerlach M, Double K, Arzberger T, Leblhuber F, Tatschner T, Riederer P (2003). Dopamine receptor agonists in current clinical use: comparative dopamine receptor binding profiles defined in the human striatum. J Neural Transm 110: 1119-1127.

Gotham AM, Brown RG, Marsden CD (1988). Frontal' cognitive function in patients with Parkinson's disease 'on' and 'off levodopa. Brain 111: 299-321.

Graef S, Biele G, Krugel LK, Marzinzik F, Wahl M, Wotka J et al (2010). Differential influence of levodopa on reward-based learning in Parkinson's disease. Front Hum Neurosci 4: 169.

Hoehn MM, Yahr MD (1967). Parkinsonism: onset, progression and mortality. Neurology 17: 427-442.

Housden CR, O'Sullivan SS, Joyce EM, Lees AJ, Roiser JP (2010). Intact reward learning but elevated delay discounting in Parkinson's disease patients with impulsive-compulsive spectrum behaviors. Neuropsychopharmacology 35: 2155-2164.

Hughes AJ, Daniel SE, Kilford L, Lees AJ (1992). Accuracy of clinical diagnosis of idiopathic Parkinson's disease: a clinicopathological study of 100 cases. J Neurol Neurosurg Psychiatry 55: $181-184$.

Ishibashi K, Ishii K, Oda K, Mizusawa H, Ishiwata K (2011). Binding of pramipexole to extrastriatal dopamine D2/D3 receptors in the human brain: a positron emission tomography study using 11C-FLB 457. PLoS One 9: e17723.

Jahanshahi M, Wilkinson L, Gahir H, Dharminda A, Lagnado DA (2010). Medication impairs probabilistic classification learning in Parkinson's disease. Neuropsychologia 48: 1096-1103.

Kapur S (2003). Psychosis as a state of aberrant salience: a framework linking biology, phenomenology, and pharmacology in schizophrenia. Am J Psychiatry 160: 13-23.

King R, Barchas JD, Huberman BA (1984). Chaotic behavior in dopamine neurodynamics. Proc Natl Acad Sci USA 81: 1244-1247.

Kish SJ, Shannak K, Hornykiewicz O (1988). Uneven pattern of dopamine loss in the striatum of patients with idiopathic 
Parkinson's disease. Pathophysiologic and clinical implications. $N$ Engl J Med 318: 876-880.

Knutson B, Bjork JM, Fong GW, Hommer D, Mattay VS, Weinberger DR (2004). Amphetamine modulates human incentive processing. Neuron 43: 261-269.

Kobayakawa M, Tsuruya N, Kawamura M (2010). Sensitivity to reward and punishment in Parkinson's disease: an analysis of behavioral patterns using a modified version of the Iowa gambling task. Parkinsonism Relat Disord 16: 453-457.

Kosaka H, Omori M, Murata T, Iidaka T, Yamada H, Okada T et al (2002). Differential amygdala response during facial recognition in patients with schizophrenia: an fMRI study. Schizophr Res 57: 87-95.

Lang AE, Fahn S (1989). Assessment of Parkinson's disease. In: Munsat TL (ed) Quantification of Neurologic Deficit. Butterworth-Heinemann: Boston, Mass. pp 285-309.

MacDonald PA, Macdonald AA, Seergobin KN, Tamjeedi R, Ganjavi H, Provost JS et al (2011). The effect of dopamine therapy on ventral and dorsal striatum-mediated cognition in Parkinson's disease: support from functional MRI. Brain 134: 1447-1463.

Maia TV, Frank MJ (2011). From reinforcement learning models to psychiatric and neurological disorders. Nat Neurosci 14: 154-162.

Mannan SK, Hodgson TL, Husain M, Kennard C (2008). Eye movements in visual search indicate impaired saliency processing in Parkinson's disease. Prog Brain Res 171: 559-562.

Mason O, Claridge G, Jackson M (1995). New scales for the assessment of schizotypy. Pers Indiv Differ 18: 7-13.

McGowan S, Lawrence AD, Sales T, Quested D, Grasby P (2004). Presynaptic dopaminergic dysfunction in schizophrenia: a positron emission tomographic [18F]fluorodopa study. Arch Gen Psychiatry 61: 134-142.

Mehler-Wex C, Riederer P, Gerlach M (2006). Dopaminergic dysbalance in distinct basal ganglia neurocircuits: implications for the pathophysiology of Parkinson's disease, schizophrenia and attention deficit hyperactivity disorder. Neurotox Res 10: 167-179.

Miller R (1993). Striatal dopamine in reward and attention: a system for understanding the symptomatology of acute schizophrenia and mania. Int Rev Neurobiol 35: 161-278.

Moore JW, Schneider SA, Schwingenschuh P, Moretto G, Bhatia KP, Haggard P (2010). Dopaminergic medication boosts actioneffect binding in Parkinson's disease. Neuropsychologia 48: $1125-1132$

Mountjoy CQ, Roth M (1982). Studies in the relationship between depressive disorders and anxiety states. Part 1. Rating scales. J Affect Disord 4: 127-147.

Pessiglione M, Seymour B, Flandin G, Dolan RJ, Frith CD (2006). Dopamine-dependent prediction errors underpin reward-seeking behaviour in humans. Nature 442: 1042-1045.

Roiser JP, Stephan KE, den Ouden HE, Barnes TR, Friston KJ, Joyce EM (2009). Do patients with schizophrenia exhibit aberrant salience? Psychol Med 39: 199-209.
Roiser JP, Stephan KE, den Ouden HE, Friston KJ, Joyce EM (2010). Adaptive and aberrant reward prediction signals in the human brain. Neuroimage 50: 657-664.

Schmidt K, Roiser JP (2009). Assessing the construct validity of aberrant salience. Front Behav Neurosci 3: 58.

Schultz W (2007). Multiple dopamine functions at different time courses. Annu Rev Neurosci 30: 259-288.

Shaner A (1999). Delusions, superstitious conditioning and chaotic dopamine neurodynamics. Med Hypotheses 52: 119-123.

Shohamy D, Myers CE, Geghman KD, Sage J, Gluck MA (2006). Ldopa impairs learning, but spares generalization, in Parkinson's disease. Neuropsychologia 44: 774-784.

Stoner SC, Dahmen MM, Makos M, Lea JW, Carver LJ, Rasu RS (2009). An exploratory retrospective evaluation of ropiniroleassociated psychotic symptoms in an outpatient population treated for restless legs syndrome or Parkinson's disease. Ann Pharmacother 43: 1426-1432.

Subramanian L, Hindle JV, Jackson MC, Linden DE (2010). Dopamine boosts memory for angry faces in Parkinson's disease. Mov Disord 25: 2792-2799.

van Eimeren T, Ballanger B, Pellecchia G, Miyasaki JM, Lang AE, Strafella AP (2009). Dopamine agonists diminish value sensitivity of the orbitofrontal cortex: a trigger for pathological gambling in Parkinson's disease? Neuropsychopharmacology 34: 2758-2766.

Verbaan D, van Rooden SM, Visser M, Marinus J, Emre M, van Hilten JJ (2009). Psychotic and compulsive symptoms in Parkinson's disease. Mov Disord 24: 738-744.

Voss M, Moore J, Hauser M, Gallinat J, Heinz A, Haggard P (2010). Altered awareness of action in schizophrenia: a specific deficit in predicting action consequences. Brain 133: 3104-3112.

Voon V, Fox SH (2007). Medication-related impulse control and repetitive behaviors in Parkinson disease. Arch Neurol 64: 1089-1096.

Wechsler D (1981). Wechsler Adult Intelligence Scale-Revised Manual. Psychological Corporation: New York, NY.

Weintraub D, Burn DJ (2011). Parkinson's disease: the quintessential neuropsychiatric disorder. Mov Disord 26: 1022-1031.

Winstanley CA, Cocker PJ, Rogers RD (2011). Dopamine modulates reward expectancy during performance of a slot machine task in rats: evidence for a 'near-miss' effect. Neuropsychopharmacology 36: 913-925.

Wyvell CL, Berridge KC (2000). Intra-accumbens amphetamine increases the conditioned incentive salience of sucrose reward: enhancement of reward 'wanting' without enhanced 'liking' or response reinforcement. J Neurosci 20: 8122-8130.

Ye Z, Hammer A, Camara E, Münte TF (2011). Pramipexole modulates the neural network of reward anticipation. Hum Brain Mapp 32: 800-811.

Yin HH, Knowlton BJ (2006). The role of the basal ganglia in habit formation. Nat Rev Neurosci 7: 464-476.

Young RC, Biggs JT, Ziegler VE, Meyer DA (1978). A rating scale for mania: reliability, validity and sensitivity. Br J Psychiatry 133: $429-435$. 